INPLASY

PROTOCOL

To cite: Zhang et al.

Complementary and alternative therapies for precancerous lesions of gastric cancer: A Bayesian network meta-analysis protocol. Inplasy protocol 2020120077. doi: 10.37766/inplasy2020.12.0077

Received: 14 December 2020

Published: 14 December 2020

Corresponding author:

Tiefeng Zhang

duanduan8525@126.com

Author Affiliation:

Second Affiliated Hospital of

Shandong University of

Traditional Chinese Medicine

Support: 2015GSF119031.

Review Stage at time of this submission: Preliminary searches.

Conflicts of interest: None.

\section{Complementary and alternative therapies for precancerous lesions of gastric cancer: A Bayesian network meta-analysis protocol}

\author{
Zhang, TQ1; Zhang, TF2; Li, CQ3; Zhai, XX4; Huo, Q55.
}

Review question / Objective: To get high-quality evidence for making scientific decisions, we carry out a Bayesian NMA to compare and rank different complementary and alternative therapies for the patients with Precancerous lesion of gastric cancer (PLGC).

Condition being studied: Precancerous lesion of gastric cancer (PLGC) is considered to be a crucial phase in the early diagnosis and prevention of intestinal gastric cancer. It takes a relatively long process for PLGC to develop into gastric cancer. Due to active follow-up monitoring and effective intervention, the development of gastric cancer is blocked, and the incidence and mortality of gastric cancer will be significantly reduced. Modern medical examination methods play an important role in the diagnosis of PLGC and early detection of lesions can be achieved. However, there is no ideal intervention and treatment measures for PLGC, and drug treatment is still controversial. Thus, complementary and alternative therapies are getting more and more attention. In recent years, complementary and alternative therapies have achieved prominent clinical effects in treating PLGC, which mainly include Chinese herbal medicine, acupuncture, massage, acupoint injection, psychotherapy, exercise therapy and so on.

INPLASY registration number: This protocol was registered with the International Platform of Registered Systematic Review and Meta-Analysis Protocols (INPLASY) on 14 December 2020 and was last updated on 14 December 2020 (registration number INPLASY2020120077).

\section{INTRODUCTION}

Review question / Objective: To get highquality evidence for making scientific decisions, we carry out a Bayesian NMA to compare and rank different complementary and alternative therapies for the patients with Precancerous lesion of gastric cancer (PLGC). 
Condition being studied: Precancerous lesion of gastric cancer (PLGC) is considered to be a crucial phase in the early diagnosis and prevention of intestinal gastric cancer. It takes a relatively long process for PLGC to develop into gastric cancer. Due to active follow-up monitoring and effective intervention, the development of gastric cancer is blocked, and the incidence and mortality of gastric cancer will be significantly reduced. Modern medical examination methods play an important role in the diagnosis of PLGC and early detection of lesions can be achieved. However, there is no ideal intervention and treatment measures for PLGC, and drug treatment is still controversial. Thus, complementary and alternative therapies are getting more and more attention. In recent years, complementary and alternative therapies have achieved prominent clinical effects in treating PLGC, which mainly include Chinese herbal medicine, acupuncture, massage, acupoint injection, psychotherapy, exercise therapy and so on.

\section{METHODS}

Participant or population: Patients who meet the diagnostic criteria of precancerous lesion of gastric cancer (PLGC) ; There is no restrictions in gender, age, race and nationality. Patients with other complicated and severe diseases will not be included.

Intervention: PLGC patients in the treatment group are given complementary and alternative therapies, which include Chinese herbal medicine, acupuncture, massage, acupoint injection, psychotherapy, exercise therapy and so on. Different complementary and alternative therapies can be freely combined. In addition, they can be adopted based on routine western medicine or separately.

Comparator: The control group will adopt conventional western medicine, placebo, or other positive drugs. There is no restriction on the dosage form.
Study designs to be included: This study will contain all relevant peer-reviewed randomized controlled trials(RCTs). Review, animal study, case report, conference paper will not be included. The sample size of both treatment group and control group is at least 30 . The language will be confined to Chinese or English.

Eligibility criteria: 1 . Type of study: This study will contain all relevant peerreviewed randomized controlled trials (RCTs). Review, animal study, case report, conference paper will not be included. The sample size of both treatment group and control group is at least 30 . The language will be confined to Chinese or English. 2. Participants: Patients who meet the diagnostic criteria of precancerous lesion of gastric cancer (PLGC) ; There is no restrictions in gender, age, race and nationality. Patients with other complicated and severe diseases will not be included. 3 . Interventions: PLGC patients in the treatment group are given complementary and alternative therapies, which include Chinese herbal medicine, acupuncture, massage, a cupoint injection, psychotherapy, exercise therapy and so on. Different complementary and alternative therapies can be freely combined. In addition, they can be adopted based on routine western medicine or separately. 4 . Comparisons: The control group will adopt conventional western medicine, placebo, or other positive drugs. There is no restriction on the dosage form. 5. Outcomes: The main outcomes: clinical efficacy rate, Helicobacter pylori infection clearance rate; Secondary outcomes: the improvement rate of gastroscopy, the improvement rate of gastric mucosa pathology, Traditional Chinese Medicine Syndrome Score Scale (TCMSSS), the incidence of adverse events, economic costs.

Information sources: Databases and search strategy:We will carefully discuss the details and precautions about literature retrieval, and then ascertain the search strategy after pre-retrieving literature. We 
will utilize a combination of MeSH and freetext terms. The retrieval time is from the establishment of the database to December 2020. All patients qualified in the trial are diagnosed as PLGC by clinical and endoscopic pathology. Search databases are as follows: PubMed, Cochrane Library, Cochrane Controlled Trial Center Registration, EMBASE, Web of Science, CNKI, Wanfang database, VIP database. In addition, we will proceed to follow up the literature in systematic review / metaanalysis. The search scheme of PubMed database is shown in Table 1. Data extraction:On the basis of the above strategy, we will retrieve all relevant literature from the database, and then import them into EndNote X9. Two researchers (Tianqi Zhang and Xixi Zhai) will browse the title and abstract separately, sort out the literature according to the inclusion and exclusion criteria, and then extract the data. Based on the previous step, qualified literature will be further searched. If the two researchers have different opinions, they will discuss and solve them or hand over to the third researcher (Chuancheng $\mathrm{Li}$ ) for assistance. If the collected literature is incomplete, we will attempt to consult the corresponding author for help.

Main outcome(s): Clinical efficacy rate and Helicobacter pylori infection clearance rate.

Additional outcome(s): The improvement rate of gastroscopy, the improvement rate of gastric mucosa pathology, Traditional Chinese Medicine Syndrome Score Scale (TCMSSS), the incidence of adverse events, economic costs, etc

Quality assessment / Risk of bias analysis: The methodological quality will be evaluated by two researchers independently in the light of the Cochrane Collaboration's Risk of Bias Tool , which contains 7 items and every item is classified as "high" "unclear" and "low" levels.

Strategy of data synthesis: 1.Pairwise meta-analysis:We will execute STATA16.0A for pairwise meta-analysis. Continuous results will be summarized by SMD with $95 \% \mathrm{Cl}$ while dichotomous results will be analyzed by OR with $95 \% \mathrm{Cl}$. 12 will be adopted to evaluate the heterogeneity of each pairwise comparisons. 2.NMA:NMA will be implemented by STATA 16.0 and WinBUGS1.4.3 to synthesize direct and indirect evidence. The Bayesian NMA is mainly on the basis of the Markov-chainMonte-Carlo (MCMC), so we will utilize the MCMC in WinBUGS1.4.3 to execute Bayesian NMA of random effect model[18]. After statistical analysis, the potential scale reduction factor (PSRF) is used to measure the convergence. When it is closer to 1 , the convergence is much better, and the conclusion is more reliable. Besides, we will adjust iteration times and annealing times based on the actual situation, and estimate the corresponding effective value of $95 \% \mathrm{Cl}$. Moreover, this study will adopt surface under the cumulative ranking curve values to predict and rank the order of therapeutic effect. If the area under the curve is larger, the therapeutic effect is better. Furthermore, we will analyze the consistency of the main indicators. Suppose there is a closed loop, we will apply the node splitting method to estimate each loop's inconsistency.

Subgroup analysis: Considering the heterogeneity, we will perform subgroup analysis in the light of the characteristics of research related to the source of heterogeneity. In addition, with regard to different design schemes, we will adopt subgroup analysis in accordance with age, gender, treatment type and course of disease.

Sensibility analysis: Sensitivity analysis will be assessed by excluding the literature one by one to ascertain whether the literature has an impact on heterogeneity. Once the heterogeneity of the study changes, this article may be the source of heterogeneity, and further analysis will be carried out, such as the difference in sample size and the reference standard of outcome indicators. If there is no obvious change before and after exclusion, it indicates that the results are stable and credible. 
Country(ies) involved: China.

Keywords: precancerous lesion of gastric cancer, Bayesian, network meta-analysis, complementary and alternative therapies, protocols.

Contributions of each author:

Author 1 - Tianqi Zhang.

Author 2 - Tiefeng Zhang.

Author 3 - Chuancheng Li.

Author 4 - Xixi Zhai.

Author 5 - Qing Huo. 